\title{
Biomarkers for the prediction of oncologic outcomes in non- muscle invasive bladder cancer: state of affairs and new frontiers
}

\author{
Francesco Soria $^{1,2}$, Shahrokh F. Shariat ${ }^{1,3,4,5}$ \\ ${ }^{1}$ Department of Urology and Comprehensive Cancer Center, Medical University of Vienna, Vienna, Austria; ${ }^{2}$ Division of Urology, Department \\ of Surgical Sciences, San Giovanni Battista Hospital, University of Studies of Torino, Turin, Italy; ${ }^{3}$ Karl Landsteiner Institute of Urology and \\ Andrology, Vienna, Austria; ${ }^{4}$ Department of Urology, Weill Cornell Medical College, New York Presbyterian Hospital, New York, NY, USA; \\ ${ }^{5}$ Department of Urology, University of Texas Southwestern Medical Center, Dallas, USA \\ Correspondence to: Shahrokh F. Shariat. Department of Urology and Comprehensive Cancer Center, Vienna General Hospital, Medical University of \\ Vienna, Währinger Gürtel 18-20, A-1090 Vienna, Austria. Email: shahrokh.shariat@meduniwien.ac.at. \\ Provenance: This is an Invited Editorial commissioned by Section Editor Xiao Li (Department of Urology, Jiangsu Cancer Hospital \& Jiangsu \\ Institute of Cancer Research \& Nanjing Medical University Affiliated Cancer Hospital, Nanjing, China). \\ Comment on: van Kessel KEM, van der Keur KA, Dyrskjøt L, et al. Molecular Markers Increase Precision of the European Association of Urology \\ Non-Muscle-Invasive Bladder Cancer Progression Risk Groups. Clin Cancer Res 2018;24:1586-93.
}

Submitted Aug 03, 2018. Accepted for publication Aug 07, 2018.

doi: $10.21037 /$ tau. 2018.08 .10

View this article at: http://dx.doi.org/10.21037/tau.2018.08.10

\section{Introduction}

Non-muscle invasive bladder cancer (NMIBC) represents approximately $75 \%$ of newly diagnosed bladder cancers (BCa) in western countries (1). Patients with NMIBC have a relatively favorable prognosis, with ten-years cancerspecific survival (CSS) rates varying between $75 \%$ and $100 \%$, depending on tumor grade (2). Nevertheless, despite adequate therapy, patients with NMIBC have a life-long risk of disease recurrence and, more importantly, of progression to muscle-invasive bladder cancer (MIBC) (3). While cancer recurrence mainly impacts our patients' quality of life and the economical burden of the disease, progression to $\mathrm{MIBC}$ represents a dramatic event, significantly lowering survival probability and calling for intensified therapy such as radical cystectomy (4). Indeed, patients harboring a NMIBC that eventually progress to MIBC have a worse survival probability compared to a patient who presents with a primary MIBC (5). Because of these reasons, predicting both disease recurrence and progression is of fundamental importance to accurately stratify patients into personalized risk groups for selection of the appropriate treatment strategy, which can range from variable follow up scheduling, from adjuvant intravesical therapy schemes to radical surgery. A personalized assessment of the biologic potential and clinical behavior of NMIBC in every specific patient could allow for an improvement of oncologic outcomes and smart allocation of resources.

\section{Status quo in the prediction of outcomes in NMIBC}

Currently, risk-stratification of patients with NMIBC is based on patients' characteristics and tumor-related features. Based on tumor stage, grade, presence of carcinoma in situ (CIS), tumor size, tumor number and previous recurrence rate, the European Organization for Research and Treatment of Cancer (EORTC) risk tables stratify patients into low, intermediate and high risk for each disease recurrence and progression (6). Since these risk tables were built using clinical trial data of patients treated in previous decades before the wide spread use of BCG immunotherapy and re-TUR, their predictive accuracy is limited in contemporary patients. The Spanish Urological Club for Oncological Treatment (CUETO) group tried to overcome these limitations by including only patients treated with BCG and by adding additional features to the model such as age and gender (7). However, the discrimination of even this nomogram remains unsatisfactory when tested in external validation cohorts (8). Both tools exhibited poor discrimination for both disease recurrence and progression 
(0.60 and 0.66 , and 0.52 and 0.62 , for the EORTC and CUETO models, respectively), underlying the need for better tools incorporating more powerful predictors of oncologic behavior in order to improve NMIBC riskstratification and therapy.

One hope is to fill the "missing information" by integrating biomarkers that reflect the biological behavior of the cells and its host thereby increasing the capture of the tumors personality. To date, several urinary, blood and tissue markers have been developed and tested with the aim to improving prediction of outcomes and helping with selection, thereby moving a step forward towards the era of personalized medicine. However, due to their suboptimal performances, their role remains, as of today, still limited and none of them is currently recommended by expert guidelines for daily clinical practice (9).

Urinary biomarkers have been used to predict short to intermediate term oncological outcomes as well as response to BCG. A positive fluorescence in situ hybridization (FISH) assay, for example, performed at different time points during BCG therapy, was associated with either disease persistence or recurrence $(10,11)$. Kamat et al. found that a positive FISH both at 6 and 12 weeks resection on BCG therapy can identify patients at higher risk of disease recurrence and progression (12). While promising, validations of these findings are still pending.

Blood-based biomarkers measuring systemic inflammatory response such as the neutrophil-tolymphocyte ratio (NLR) and the C-reactive protein (CRP) have also been evaluated as predictors of oncological outcomes in NMIBC. Their integration into a model for the prediction of disease recurrence and progression led to an increase in the discrimination of the model (13). These biomarkers are interesting as they may be able to help patients most likely to benefit from systemic immunotherapy such as check-point inhibitors.

A growing body of literature shows that several genes and proteins related to different pathways are not only involved in bladder carcinogenesis but also in its clinical behavior. Consequently, several tissue biomarkers have been tested in a multiphased systematic approach (14). Even if multiple biomarkers, such as cell-cycle markers as well as Ki-67, FGFR3, cadherins, surviving as well as immune and inflammation-related biomarkers have shown to predict NMIBC outcomes, their prognostic value remains suboptimal with only few of them having prospective validation study phases (15-20). Recently, van Kessel et al. prospectively tested a panel of tissue biomarkers comparing their performance to current clinicopathological characteristics for risk-stratification (21). Fresh frozen tumor samples from 1,239 patients with primary or recurrent NMIBC were analyzed for GATA2, TBX2, TBX3 and ZIC4 methylation and FGFR3, TERT, PIK3CA and RAS mutation status. Overall, wild type FGFR3 and methylation of GATA2 and TBX3 were significantly associated with disease progression; the addition of these selected markers to the EORTC risk stratification model increased its accuracy and was able to identify a subset of patients at very high risk for tumor progression. This is probably clinically the most significant finding of this study, as one of the major controversies in NMIBC management is to identify the patients who are most likely to benefit from intensified therapy such as combination systemic therapy or early radical cystectomy.

\section{Conclusions}

The search for an ideal biomarker in NMIBC is still ongoing. Given the variable and rich mutation landscape, branched evolution and intratumor heterogeneity of the disease, it is unlikely that a single biomarker is able to address the diverse needs of clinicians. Conversely, biomarkers panels integrating multiple complementary pathways involved in the process of interest (diagnosis, staging, prognosis, and/or prediction) could represent a breakthrough for patients' risk stratification and treatment selection. Several new biomarkers, probably linked to novel therapies such as PD-L1 expression, will soon enter in clinical practice helping drive a precision medicine approach to $\mathrm{BCa}$. We are slowly but steadily moving towards the era of personalized medicine with biomarkers being the traffic light and/or the target of the personalized medicine voyage in NMIBC.

\section{Acknowledgements}

None.

\section{Footnote}

Conflicts of Interest: The authors have no conflicts of interest to declare.

\section{References}

1. Burger M, Catto JW, Dalbagni G, et al. Epidemiology 
and risk factors of urothelial bladder cancer. Eur Urol 2013;63:234-41.

2. Gontero P, Sylvester R, Pisano F, et al. Prognostic factors and risk groups in T1G3 non-muscle-invasive bladder cancer patients initially treated with Bacillus CalmetteGuérin: results of a retrospective multicenter study of 2451 patients. Eur Urol 2015;67:74-82.

3. Malmström PU, Sylvester RJ, Crawford DE, et al. An Individual Patient Data Meta-Analysis of the LongTerm Outcome of Randomised Studies Comparing Intravesical Mitomycin C versus Bacillus CalmetteGuérin for Non-Muscle-Invasive Bladder Cancer. Eur Urol 2009;56:247-56.

4. Soria F, Gontero P. How to assess and improve healthrelated quality of life in bladder cancer patients. Transl Androl Urol 2018;7 Suppl 1:S77-S80.

5. Moschini M, Sharma V, Dell'oglio P, et al. Comparing long-term outcomes of primary and progressive carcinoma invading bladder muscle after radical cystectomy. BJU Int 2016;117:604-10.

6. Sylvester RJ, van der Meijden AP, Oosterlinck W, et al. Predicting recurrence and progression in individual patients with stage Ta T1 bladder cancer using EORTC risk tables: a combined analysis of 2596 patients from seven EORTC trials. Eur Urol 2006;49:466-5; discussion 475-7.

7. Fernandez-Gomez J, Madero R, Solsona E, et al. Predicting nonmuscle invasive bladder cancer recurrence and progression in patients treated with bacillus Calmette-Guerin: the CUETO scoring model. J Urol 2009; 182:2195-203.

8. Xylinas E, Kent M, Kluth L, et al. Accuracy of the EORTC risk tables and of the CUETO scoring model to predict outcomes in non-muscle-invasive urothelial carcinoma of the bladder. Br J Cancer 2013;109:1460-6.

9. Babjuk M, Böhle A, Burger M, et al. EAU Guidelines on Non-Muscle-invasive Urothelial Carcinoma of the Bladder: Update 2016. Eur Urol 2017;71:447-61.

10. Liem EI, Baard J, Cauberg EC, et al. Fluorescence in situ hybridization as prognostic predictor of tumor recurrence during treatment with Bacillus Calmette-Guérin therapy for intermediate- and high-risk non-muscle-invasive bladder cancer. Med Oncol 2017;34:172.

11. Savic S, Zlobec I, Thalmann GN, et al. The prognostic value of cytology and fluorescence in situ hybridization in the follow-up of nonmuscle-invasive bladder cancer after intravesical Bacillus Calmette-Guérin therapy. Int J Cancer 2009;124:2899-904.

12. Soria F, Droller MJ, Lotan Y, et al. An up-to-date catalog of available urinary biomarkers for the surveillance of nonmuscle invasive bladder cancer. World J Urol 2018. [Epub ahead of print].

13. Mbeutcha A, Shariat SF, Rieken M, et al. Prognostic significance of markers of systemic inflammatory response in patients with non-muscle-invasive bladder cancer. Urol Oncol 2016;34:483.e17-24.

14. Bensalah K, Montorsi F, Shariat SF. Challenges of cancer biomarker profiling. Eur Urol 2007;52:1601-9.

15. D'Andrea D, Hassler MR, Abufaraj M, et al. Progressive tissue biomarker profiling in non-muscle-invasive bladder cancer. Expert Rev Anticancer Ther 2018;18:695-703.

16. Shariat SF, Zlotta AR, Ashfaq R, et al. Cooperative effect of cell-cycle regulators expression on bladder cancer development and biologic aggressiveness. Mod Pathol 2007;20:445-59.

17. Shariat SF, Ashfaq R, Karakiewicz PI, et al. Survivin expression is associated with bladder cancer presence, stage, progression, and mortality. Cancer 2007;109:1106-13.

18. Shariat SF, Kim J, Raptidis G, et al. Association of p53 and p2 1 expression with clinical outcome in patients with carcinoma in situ of the urinary bladder. Urology 2003;61:1140-5.

19. Shariat SF, Kim JH, Ayala GE, et al. Cyclooxygenase-2 is highly expressed in carcinoma in situ and T1 transitional cell carcinoma of the bladder. J Urol 2003;169:938-42.

20. Byrne RR, Shariat SF, Brown R, et al. E-cadherin immunostaining of bladder transitional cell carcinoma, carcinoma in situ and lymph node metastases with longterm followup. J Urol 2001;165:1473-9.

21. van Kessel KEM, van der Keur KA, Dyrskjøt L, et al. Molecular Markers Increase Precision of the European Association of Urology Non-Muscle-Invasive Bladder Cancer Progression Risk Groups. Clin Cancer Res 2018;24:1586-93.

Cite this article as: Soria F, Shariat SF. Biomarkers for the prediction of oncologic outcomes in non-muscle invasive bladder cancer: state of affairs and new frontiers. Transl Androl Urol 2018;7(Suppl 6):S753-S755. doi: 10.21037/tau.2018.08.10 